\title{
X-ray and ultraviolet observations of the eclipsing cataclysmic variables OV Bootis and SDSS J103533.02+055158.3 with degenerate donors ${ }^{\star}$
}

\begin{abstract}
Axel Schwope ${ }^{1}$, Hauke Worpel ${ }^{1}$, and Iris Traulsen ${ }^{1}$
Leibniz-Institut für Astrophysik Potsdam (AIP), An der Sternwarte 16, 14482 Potsdam, Germany

January 11,2021

ABSTRACT

Context. The majority of cataclysmic variables are predicted to be post-period minimum systems with degenerate donor stars, the period bouncers. Owing to their intrinsic faintness, however, only a handful of these systems have so far been securely identified. Aims. We want to study the X-ray properties of two eclipsing period bouncers, OV Bootis and SDSS J103533.02+055158.3, that were selected for this study due to their proximity to Earth.

Methods. We have obtained XMM-Newton phase-resolved X-ray and ultraviolet observations of the two objects for spectral and timing analysis.

Results. Owing to a recent dwarf nova outburst OV Bootis was much brighter than SDSS J103533.02+055158.3 at X-ray and ultraviolet wavelengths and the eclipse could be studied in some detail. An updated eclipse ephemeris was derived. The X-rays were shown to originate close to the white dwarf, the boundary layer, with significant absorption affecting its spectrum. There was no absorption in SDSS J103533.02+055158.3, despite being observed at the same inclination indicating different shapes of the disk and the disk rim. The white-dwarf temperature was re-determined for both objects: the white dwarf in OV Bootis was still hot $(23,000 \mathrm{~K})$ five months after a dwarf nova outburst, and the white dwarf in SDSS J103533.02+055158.3 hotter than assumed previously $\left(T_{\text {eff }}=11,500 \mathrm{~K}\right)$. Conclusions. All three cataclysmic variables with degenerate donors studied so far in X-rays, including SDSS J121209.31+013627.7, were clearly discovered in X-rays and revealed mass accretion rates $\dot{M} \geq 8 \times 10^{-15} \mathrm{M}_{\odot} \mathrm{yr}^{-1}$. If their X-ray behavior is representative of the subpopulation of period bouncers, the all-sky X-ray surveys with eROSITA together with comprehensive follow-up will uncover new objects in sufficient number to address the remaining questions concerning late-stage cataclysmic variable evolution.
\end{abstract}

Key words. stars: binaries: eclipsing - stars: cataclysmic variables - stars: individual: OV Bootis - stars: individual: SDSS J103533.02+055158.3 - X-rays: binaries

\section{Introduction}

Cataclysmic variables (CVs) consist of an accreting white dwarf (WD) primary and a low-mass Roche-lobe filling secondary. These systems represent the most common endpoint of close binary evolution. Below the period gap, gravitational radiation drives the period of the binary towards shorter periods. Eventually, the donor star crosses the hydrogen burning limit, at which point further mass loss causes the donor to expand. This, in turn, leads to an increase in the orbital period, and these systems are therefore referred to as period bouncers.

Although around $70 \%$ of $\mathrm{CV}$ systems are predicted to be post-period minimum (Goliasch \& Nelson 2015; Belloni et al. 2020), examples of this class have been difficult to identify owing to their intrinsic faintness. Furthermore, to distinguish between pre- and post-period minimum systems it is not enough to show that the system has a low accretion rate. The mass of the secondary must be determined to show that the donor mass is now too small to be burning hydrogen. This can be done by performing optical spectroscopy (e.g., Schwope \& Christensen 2010), or radius measurements in eclipsing systems (Burleigh et al. 2006, Littlefair et al. 2008). It is likely that many of the

* Based on observations obtained with XMM-Newton, an ESA science mission with instruments and contributions directly funded by ESA Member States and NASA secondaries in period bouncer systems are substellar in mass (Savoury et al. 2011).

To date, only a handful of unimpeachable members of the class have been identified, which were studied intensively in the optical, but little is known about their X-ray emission as a sign of accretion. Here we present an X-ray study of two confirmed period bouncers: OV Bootis and SDSS J103533.02+055158.4 (hereafter OV Boo and SDSSJ1035, respectively). These two systems were predicted to be the most X-ray bright of the known period bouncers simply due to their vicinity $(160 \pm 10 \mathrm{pc}$ for OV Boo and $171 \pm 10$ pc for SDSSJ1035; Littlefair et al. 2007. 2008 respectively) and were proposed to be observed by XMMNewton.

OV Boo was identified as a CV from Sloan Digital Sky Survey (SDSS) spectra, and follow-up observations showed it to be a deeply eclipsing system with a $1.12 \mathrm{hr}$ period (Szkody et al. 2005). The period was refined to $66.61194 \pm 0.00004$ minutes through intensive photometry by Littlefair et al. (2007) and Patterson et al. (2008). The orbital period of OV Boo lies below the period minimum predicted by standard CV evolution, and could be explained if the system were formed directly from a detached WD-brown dwarf pair. The detailed observational studies performed by Patterson et al. (2008) and Littlefair et al. (2007. 2008) revealed a complex eclipse structure, which was resolved into the WD, the accretion disk, a bright spot on the rim of the 
disk, and the donor star. Littlefair et al. (2007) also derived parameters of the WD; they give a radius of $0.0091 \pm 0.0001 R_{\odot}$ and a mass of $0.090 \pm 0.001 \mathrm{M}_{\odot}$, which we use in this paper.

From its high estimated proper motion of $\sim 160 \mathrm{mas} \mathrm{yr}^{-1}$ it is believed to be a member of the Galactic halo rather than the disk (Patterson et al. 2008). This interpretation is supported by ultraviolet (UV) observations (Uthas et al. 2011) that showed it to have significantly subsolar metallicity. In 2017 OV Boo underwent a dwarf nova (DN) outburst, studied intensively (Patterson et al. 2017; Tanabe et al. 2018), and may have had previous outbursts in 1906 and 1984 (Bengtsson 2017a b). The second Gaia data release gives OV Boo a distance of $210_{-6}^{+7} \mathrm{pc}$ (Gaia Collaboration et al.2018, Bailer-Jones et al.2018) and contrrms a high proper motion of the object of $155{\mathrm{mas} \mathrm{yr}^{-1}}^{\text {. }}$.

SDSSJ1035 has a period of 82.10 minutes, and the donor mass is $M=0.052 \pm 0.002 M_{\odot}$ (Littlefair et al. 2006). Its distance is $209_{-14}^{+16}$ pc (Gaia Collaboration et al. 2016, 2018, Bailer-Jones et al. 2018). The optical spectrum is dominated by the WD. The companion, fainter than L0, is completely undetectable (Southworth et al. 2006). Owing to the faintness of the source, little else is known about it.

We note that the Gaia distances of both objects, which we use in this paper, are larger than originally estimated.

\section{Observations and methods}

\subsection{XMM-Newton}

XMM-Newton observed OV Boo for $20.2 \mathrm{ks}$ on 2017 August 3 (ObsID 0804620201). The EPIC instruments (Strüder et al. 2001; Turner et al.2001) were operated in full-frame mode with the thin filter. The optical monitor (OM; Mason et al. 2001) observed the target in fast mode with the UVW1 filter. Effective wavelengths for the OM filters are given in Kirsch et al. (2004). The Reflection Grating Spectrometer (RGS; den Herder et al. 2001) data were taken in spectroscopy mode, but owing to the faintness of the source we have not used these data. This observation was free of high background flaring, except for one short interval near the beginning of the exposure. We obtained 1,565 photons with the EPIC-pn camera.

The same instrumental setups were used for the $23.8 \mathrm{ks} \mathrm{ob}-$ servation of SDSSJ1035 (2017 May 13, ObsID 0804620101) except that the EPIC instruments were in Small Window mode. This observation was completely unaffected by flaring.

We reduced the data with the XMM-Newton Science Analysis (SAS) software, version 16.1.0. We extracted event lists for the EPIC instruments using the emproc and epproc scripts, and corrected their timings to the Solar System barycenter using the barycen task. The OM data were reduced using the omfchain script and likewise barycenter corrected.

To extract X-ray spectra, we used circular source extraction regions centered on the target and large circular background regions containing empty sky. The spectra were grouped to 25 counts per bin, and the data from all three EPIC instruments were fitted jointly using version 12.9.1n of Xspec (Arnaud 1996).

\subsection{Swift}

SDSS1035 was observed by the Neil Gehrels Swift Observatory (Burrows et al. 2005) on 2012 July 11 (ObsID 00045684001) and 2018 October 19 (ObsID 00045684002). The X-Ray Telescope (XRT) observed in photon counting mode and the Ultraviolet Optical Telescope (UVOT) was operated in imaging mode and obtained images with the V, B, U, UVW1, UVM2, and
UVW2 filters in both observations. We did not use data from the Burst Alert Telescope because the source was too faint.

We reduced the X-ray data with the xrtpipeline task and corrected the photon arrival times to the Solar System barycenter using the barycorr task of Heasoft (Blackburn 1995). The UVOT data were reduced with the standard data reduction tasks described in the UVOT user's guide ${ }^{1}$.

There are several additional observations taken by Swift of OV Boo. Three were reported previously in Reis et al. (2013), but another eight, taken during and shortly after the outburst, have not yet been published. We present an analysis of the previously unpublished observations, which were performed in a variety of observing modes and UVOT filters, summarized in Table 1

\subsection{Chandra}

The Chandra X-ray satellite observed OV Boo serendipitously on 2003 March 14 (ObsID 4071). The second Chandra source catalog lists the object with a flux of $(8 \pm 2) \times 10^{-14} \mathrm{erg} \mathrm{cm}^{-2} \mathrm{~s}^{-1}$. Since we had a specific spectral model for which we wanted to get a flux from Chandra, we reduced the data with the ciao software package. As for XMM-Newton and Swift we extracted source photons from a circular region containing OV Boo and background photons from a larger circular source-free region.

\subsection{Optical observations}

OV Boo has almost 160,000 observations performed by the American Association of Variable Star Observers (AAVSO). We downloaded these data and corrected their timings to the Solar System barycenter using the built-in routines of Astropy, and used data that were obtained close in time to our observations with XMM-Newton to update the eclipse ephemeris.

We note that more archival data are available, namely from the Zwicky Transient Facility (ZTF; Masci et al. 2019) and the Catalina Sky Survey (CSS; Drake et al. 2009). The ZTF lists 291 measurements in both the $g$ and $r$ filters, and 15 in the $i$ filter obtained between 2018 March to 2019 June, and the CSS has 239 observations in white light obtained between April 2007 and September 2013. These data are not further analysed or used in the current paper, but we show the phase-folded light curves in the Appendix for completeness (Figures A1 and A2). The ZTF data show that OV Boo has completely recovered from the outburst in 2018 and its light curve has re-attained the shape it had at the time of the initial photometric studies by Littlefair et al. (2007) and Patterson et al. (2008) with pronounced orbital variability and a bright spot on the accretion disk rim.

\section{Analysis and results: OV Bootis}

\subsection{Orbital light curves and revised eclipse ephemeris}

In Figure 1 we show the long-term optical AAVSO and ZTFgreen light curve of OV Boo from 2016 April to 2018 July. The times of the X-ray observations are indicated by the vertical lines, red for Swift just after the peak of the eruption and blue for XMM-Newton in 2017 August after OV Boo had returned to near quiescence brightness. Prior to its eruption in 2017 the object had only been sparsely observed by AAVSO. Many observations were taken at peak and during decay. Vertical features

\footnotetext{
1 https://swift.gsfc.nasa.gov/analysis/UVOT_swguide_ v2_2.pdf
} 
Table 1. X-ray observation log

\begin{tabular}{|c|c|c|c|c|c|}
\hline Target & Inst. & Date & ObsID & $\operatorname{Exp}(\mathrm{ks})$ & Camera-Mode-Filter \\
\hline OV Boo & Chandra & 2003-Mar-14 & 4071 & 4.9 & \\
\hline \multirow[t]{2}{*}{ OV Boo } & Swift & 2017-Mar-16 & 00035443004 & 1.0 & XRT-PC \\
\hline & & & & & UVOT-IM-UVW1 \\
\hline \multirow[t]{2}{*}{ OV Boo } & Swift & 2017-Mar-18 & 00035443005 & 1.0 & XRT-PC \\
\hline & & & & & UVOT-IM-UVW1 \\
\hline \multirow[t]{2}{*}{ OV Boo } & Swift & 2017-Mar-19 & 00035443006 & 1.0 & XRT-PC, XRT-WT \\
\hline & & & & & UVOT-IM-UVW1 \\
\hline \multirow[t]{2}{*}{ OV Boo } & Swift & 2017-Mar-21 & 00035443007 & 0.6 & XRT-PC \\
\hline & & & & & UVOT-IM-UVW2, UVOT-EV-UVW2 \\
\hline \multirow[t]{2}{*}{ OV Boo } & Swift & 2017-Mar-23 & 00035443008 & 0.9 & XRT-PC \\
\hline & & & & & UVOT-EV-UVM2 \\
\hline \multirow[t]{2}{*}{ OV Boo } & Swift & 2017-Mar-24 & 00035443009 & 0.4 & XRT-PC \\
\hline & & & & & UVOT-EV-UVM2 \\
\hline \multirow[t]{2}{*}{ OV Boo } & Swift & 2017-Mar-25 & 00035443010 & 0.9 & XRT-PC \\
\hline & & & & & UVOT-EV-UVM2 \\
\hline \multirow[t]{2}{*}{ OV Boo } & Swift & 2017-Mar-28 & 00035443011 & 0.4 & XRT-PC \\
\hline & & & & & UVOT-EV-UVM2 \\
\hline \multirow[t]{2}{*}{ OV Boo } & $\mathrm{XMM}-$ Newton & 2017-Aug-03 & 0804620201 & 20.2 & EPIC-FF-THN \\
\hline & & & & & OM-FAST-UVW1 \\
\hline \multirow[t]{2}{*}{ SDSSJ1035 } & Swift & 2012-Jul-11 & 00045684001 & 3.0 & XRT-PC \\
\hline & & & & & UVOT-IM-V,B,U,UVW1,UVM2,UVW2 \\
\hline \multirow[t]{2}{*}{ SDSSJ1035 } & XMM-Newton & 2017-May-13 & 0804620101 & 23.8 & XRT-SW-THN \\
\hline & & & & & OM-FAST-UVW1 \\
\hline \multirow[t]{2}{*}{ SDSSJ1035 } & Swift & 2018-Oct-19 & 00045684002 & 1.2 & XRT-PC \\
\hline & & & & & UVOT-IM-V,B,U,UVW1,UVM2,UVW2 \\
\hline
\end{tabular}

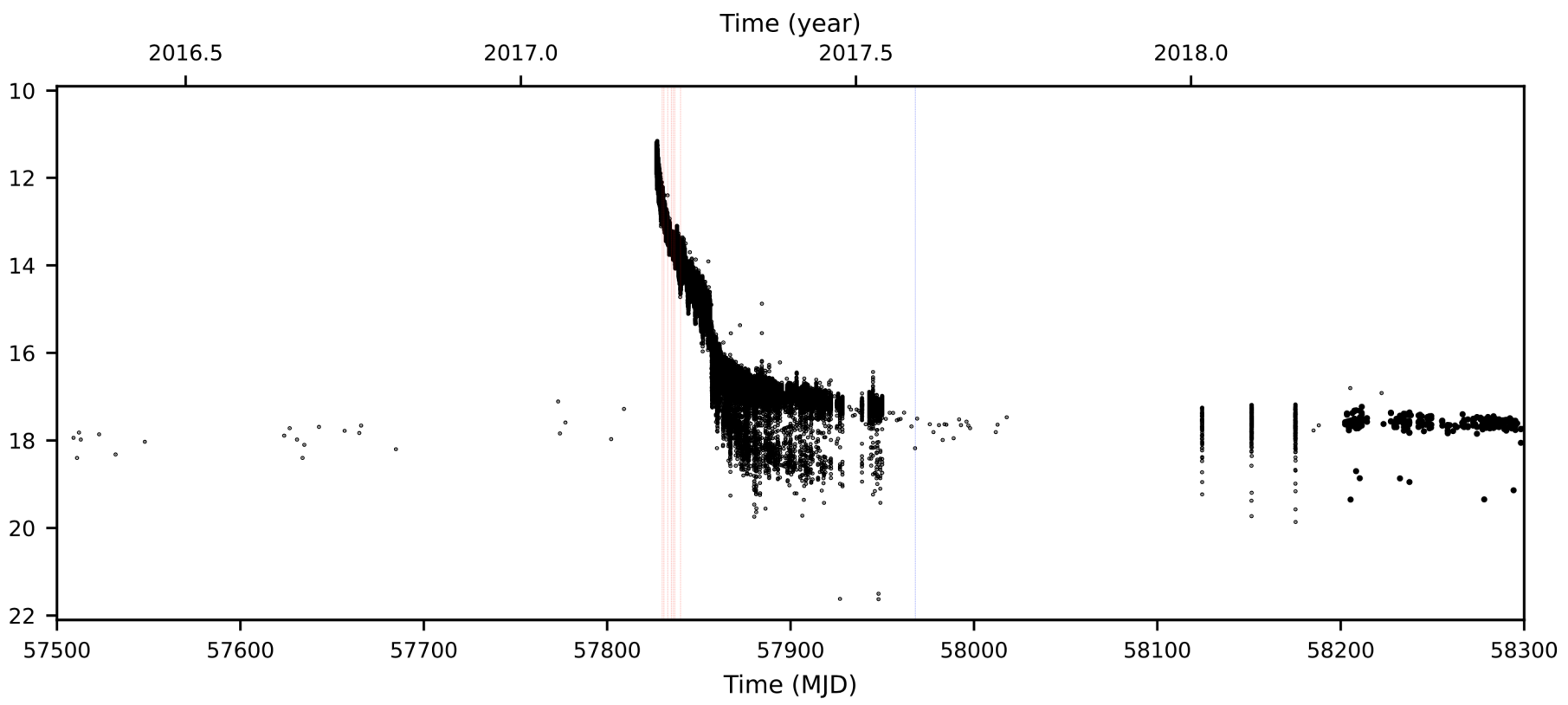

Fig. 1. Long-term optical light curve of OV Boo from 2016 April to 2018 July. AAVSO data in green, visual, or white light extend to about MJD 58200. Thereafter the larger points are ZTF g-band data. The vertical red and blue lines indicate the times of the Swift and XMM-Newton observations, respectively.

in the AAVSO data, for instance the three vertical lines between MJD 58100 and 58200, show efforts by the observers to cover the eclipses. The apparent bifurcation in the light curve beginning at about MJD 57870 also shows special attention being paid to the eclipses. Prior to the outburst OV Boo appeared to have been brightening gradually, increasing by about half a magnitude over several months.

Our new observations performed with XMM-Newton happened about 5 months after the large outburst of the source when the optical brightness had relaxed to a typical bright-phase level of $V \simeq 17.2$, still roughly one mag brighter than during quiescence. All data in this paper were transformed from observed times to orbital phase.

The original OV Boo ephemeris for the eclipse center of Patterson et al. (2008),

$H J D=2453498.892264(9)+0.0462583411(7) \times E$, 


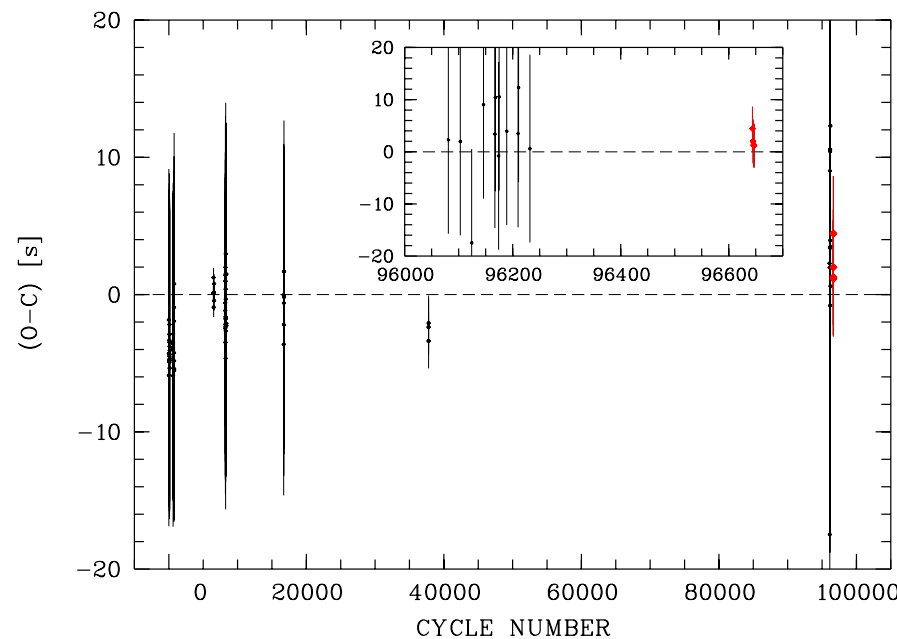

Fig. 2. Observed minus calculated mid-eclipses times for OV Boo with respect to the ephemeris given in Eq. 2 The inset shows data obtained between cycles 96000 and 96700 by AAVSO (black) and with the OM (red).

has an accumulated uncertainty of $6 \mathrm{~s}$ at the epoch of our observations with XMM-Newton and needed an update.

To produce a new mid-eclipse ephemeris for OV Boo, we took the existing mid-eclipse timings of Patterson et al. (2008) and Littlefair et al. (2007) (we used the timings derived with the $r^{\prime}$ filter in the latter paper for the documented higher accuracy) and converted them from heliocentric to barycentric timings using Astropy. The first eclipse in the data set marks the epoch for the updated ephemeris.

There were three eclipses in the Hubble Cosmic Origins Spectrograph (COS) data reported by Uthas et al. (2011). We downloaded these data, barycenter corrected the timings, and produced a $4 \mathrm{~s}$ binned light curve over the $1425-1900 \AA$ wavelength range. We did not mask out any emission lines in these spectra. Then we determined the eclipse ingresses and egresses times using the "cursor" method described in Schwope \& Thinius (2014), which gives times at half-light between time intervals in and out of the eclipse. The light curve was displayed on a graphics screen and a graphic cursor was used to determine suitable time intervals before, after, and in the eclipse to compute mean brightness levels. The time of half-light at eclipse ingress and egress was then again determined with the cursor guided by reference lines indicating the relevant brightness levels.

Similarly, we used the UV light curves of the OM binned with $2 \mathrm{~s}$ time resolution to determine the midpoints of four eclipses. We did not use timings of the three X-ray eclipses in our XMM-Newton observations because the X-ray eclipse does not seem to trace exactly the same feature as the optical and UV observations do.

Finally, there are several eclipses in the AAVSO data. We sought events observed well after the end of the 2017 DN outburst and found sixteen, most in 2017 July and a few in 2019. Eight of these, all from 2017 July, had suitable data quality to measure the ingress and egress. The complete list of eclipses is given in Table A.1. We fitted these with a weighted linear function to derive an ephemeris for mid-eclipse:

$\mathrm{BJD}(\mathrm{TDB})=2453498.8930334(34)+0.04625833911(22) \times E$

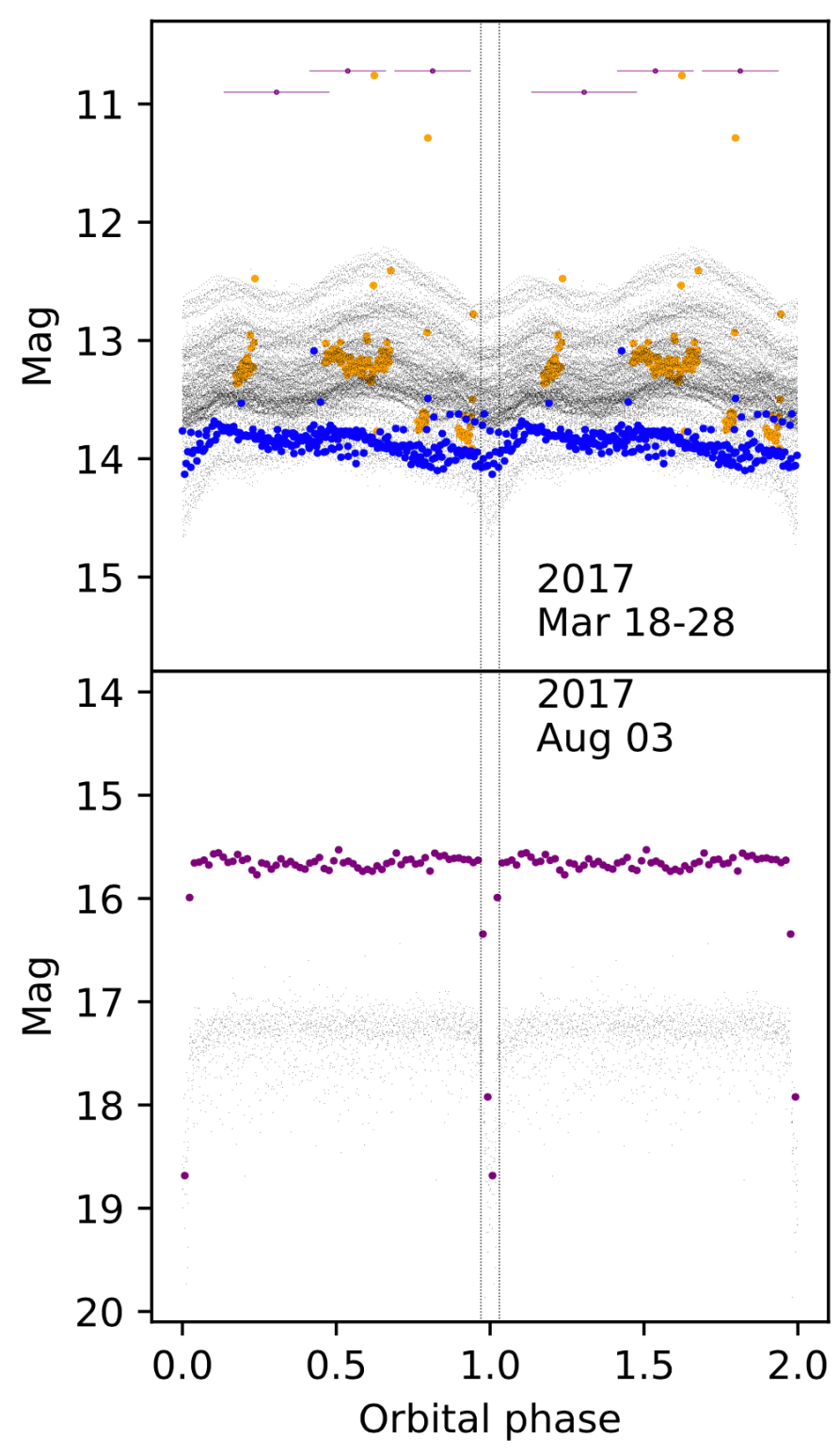

Fig. 3. Optical and UV light curves of OV Boo. Top panel: AAVSO visual (black), AAVSO B-band (blue), Swift UVOT M2 (orange), and UVOT W1 (purple). Bottom panel: XMM-Newton OM W1, binned to $64 \phi$ intervals (purple) and AAVSO visual (black). OM magnitudes are in the AB system; those from AAVSO are Vega-mags.

Values in parentheses give the uncertainties in the last digits. The residuals are also listed in Table A.1 (in the Appendix) and displayed in Fig. 2. The $\chi_{v}^{2}$ of the fit is only 0.26 (for 80 degrees of freedom), which indicates that the errors were often estimated too conservatively (too big), but we made no attempt to re-adjust the published error bars. We assumed typical error bars for our analysis of half the bin width of the used data. The ephemeris given in Eq. 2 is used to calculate phases of OV Boo throughout this paper. There is no indication of a significant deviation from linearity in the $(O-C)$ times although the most recent data obtained with the OM in the near UV and optically by AAVSO seem to occur later than predicted by our linear ephemeris. An offset of the eclipse of 2 seconds was accounted for when analyzing and modeling the UV eclipse data (see below).

We also note a slight discrepancy in the eclipse length. For the $\mathrm{OM}$ we determine $150.9 \pm 4.2 \mathrm{~s}$, which is discrepant at $2.5 \sigma$ with the very precise measurement made by Littlefair et al. 
Table 2. OV Boo X-ray spectral fit. Fluxes and luminosities are in the $0.2-10 \mathrm{keV}$ range and uncertainties are $1 \sigma$. Fluxes and luminosities are given for the sum of the two plasma emission components.

\begin{tabular}{ll} 
Parameter & Value \\
\hline $\mathrm{nH}$ & $0.24_{-0.06}^{+0.09} \times 10^{22} \mathrm{~cm}^{-2}$ \\
$\mathrm{kT}$ & $1.09_{-0.86}^{+5.03} \mathrm{keV}$ \\
norm & $2.0_{-2.0}^{+2.5} \times 10^{-5}$ \\
$\mathrm{kT}$ & $9.5_{-3.9}^{+33.5} \mathrm{keV}$ \\
norm & $2.9_{-0.4}^{+0.5} \times 10^{-4}$ \\
\hline Abs. Flux & $5.4 \pm 0.2 \times 10^{-13} \mathrm{erg} \mathrm{s}^{-1} \mathrm{~cm}^{-2}$ \\
Unabs. Flux & $6.9 \pm 0.2 \times 10^{-13} \mathrm{erg} \mathrm{s}^{-1} \mathrm{~cm}^{-2}$ \\
Abs. Lum & $2.8 \pm 0.2 \times 10^{30} \mathrm{erg} \mathrm{s}^{-1}$ \\
Unabs. Lum & $3.6 \pm 0.4 \times 10^{30} \mathrm{erg} \mathrm{s}^{-1}$ \\
\hline$\chi_{v}^{2}$ & $1.10(43)$
\end{tabular}

(2007) who derived $161.9 \pm 0.2 \mathrm{~s}$. This may raise the question whether the pre-outburst optical and the post-outburst UV emission have a common location, which we assume here. However, we cannot exclude some systematic measurement uncertainties here which gives a bias towards an apparently shorter eclipse length. The eclipse times were determined using some reference brightness values in the near vicinity of the eclipse where the brightness was already found to be somewhat reduced with respect to the expectations (see Fig. 3 , lower panel). Using a lower reference brightness shifts the ingress to a slightly later time and the egress to an earlier time, hence it affects the ingress and egress times in a similar manner. As a result, the eclipse length might appear too short, but the timing of the eclipse center is expected to be unaffected.

In Figure 3 we show the optical and UV behavior of OV Boo at and around the times of the Swift and XMM-Newton observations. In March the object was still fading from its outburst, a phenomenon clearly visible from the AAVSO points. It also appears significantly variable in all wavelengths sampled. By August, OV Boo was behaving very differently. In the optical it had faded to $m_{v} \sim 17.2$, but, apart from the eclipse, there is very little variability in either the optical or the UV.

In Figure 4 we show the phase-folded X-ray and UV light curves of OV Boo from the XMM-Newton observation. The data have been grouped into 40 bins, so that the eclipse occupies approximately one bin. The data are very coarse, but the eclipse and a bright phase centered around the eclipse are plainly visible at both energy ranges. The bright phase is not a very pronounced feature and is difficult to locate precisely in phase.

\subsection{X-ray spectral analysis of the bright phase}

We fitted the mean XMM-Newton X-ray spectrum of OV Boo, excluding the eclipse, with an absorbed (cold interstellar absorption) two-temperature plasma emission model (Mewe et al. 1985) plasma (i.e., tbabs*(mekal+mekal) in XSPEC), fitting all three instruments jointly between 0.2 and $10.0 \mathrm{keV}$. The results are given in Table 2 This spectrum is fairly typical of a $\mathrm{CV}$, though at a significantly lower luminosity. The spectrum and residuals are shown in Figure 5

Although the temperatures of the two plasma components are poorly constrained, we did need to include both. An attempt to fit the spectrum with just one thermal component prevented us from achieving an acceptable spectral fit with $\chi_{v}^{2}=1.28$ for 45

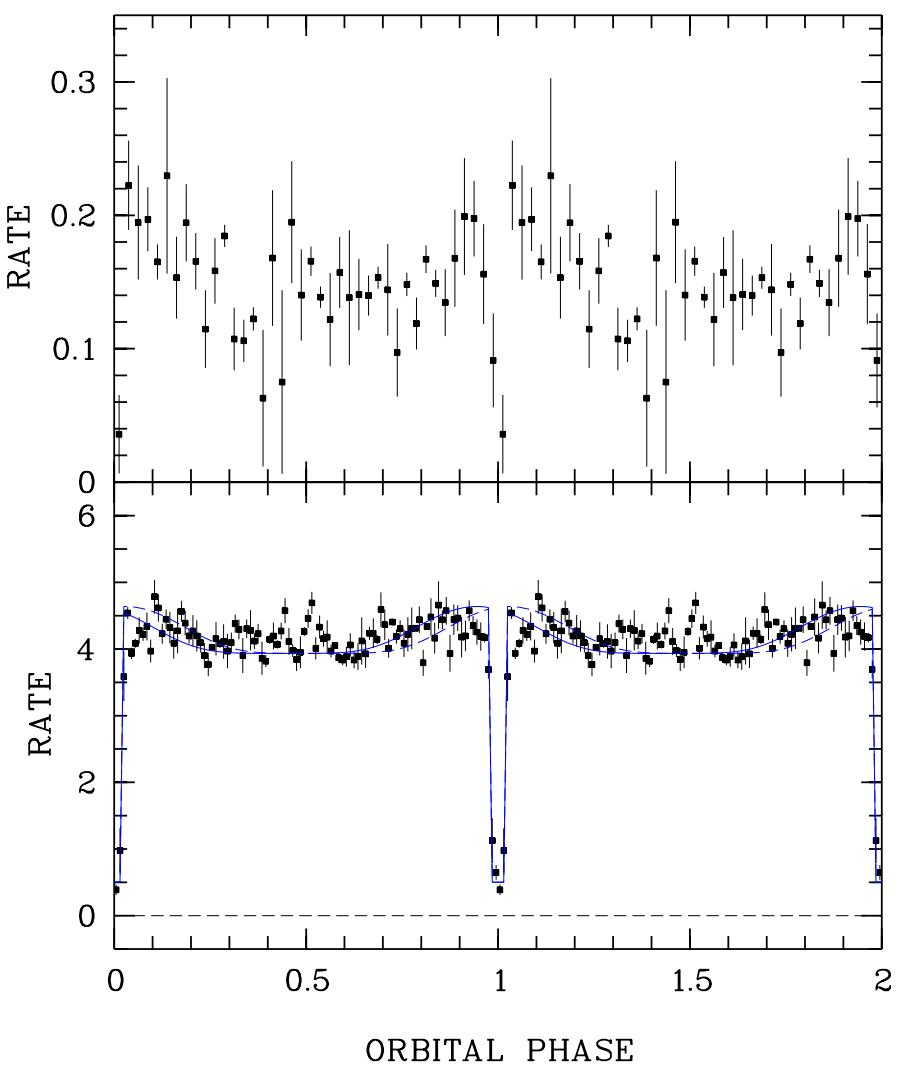

Fig. 4. Phase-folded X-ray (top panel) and UV (bottom panel) light curves of OV Boo obtained with XMM-Newton. Original time-binned data were phase-averaged using phase bins of length 0.025 and 0.01 units, which corresponds to about $100 \mathrm{~s}$ and $40 \mathrm{~s}$ at X-ray and UV wavelengths, respectively. The model curves shown in blue for two different spot locations are explained in the text.

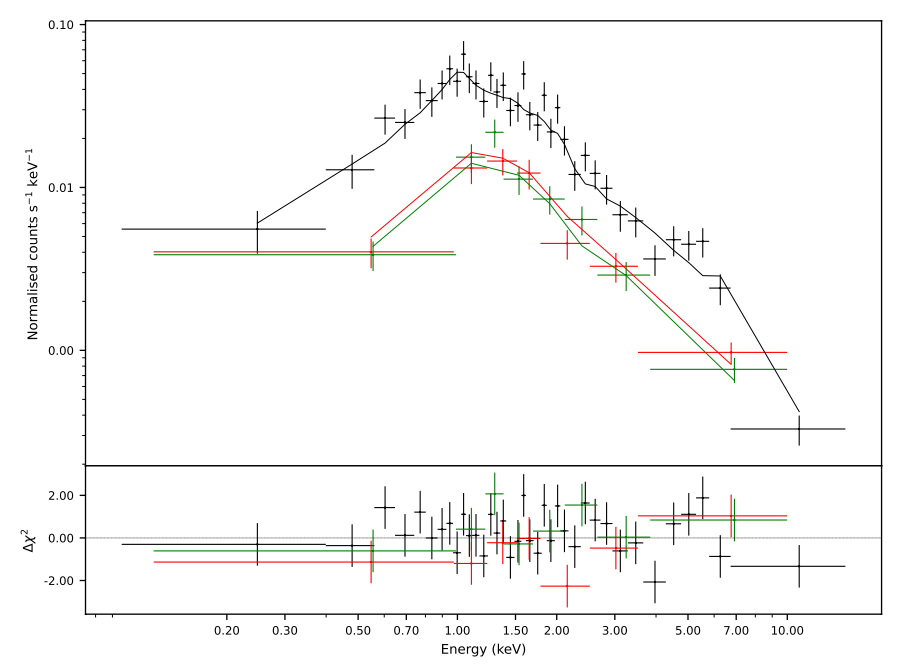

Fig. 5. X-ray spectrum of OV Boo obtained with XMM-Newton. EPIC-pn, MOS1, and MOS2 are plotted in black, red, and green, respectively, with the best fit model overplotted.

degrees of freedom. The amount of absorption we find is significantly higher than interstellar. The total column density in the direction of OV Boo is $N_{\mathrm{H}}=1.61 \times 10^{20} \mathrm{~cm}^{-2}$ (HI4PI Collaboration et al.2016), a factor of 15 lower than that found from the spectral analysis. This hints at intrinsic absorption in the binary.

We then attempted to test the predictions of Patterson et al. (2008) and Uthas et al. (2011) that OV Boo is a low-metallicity 


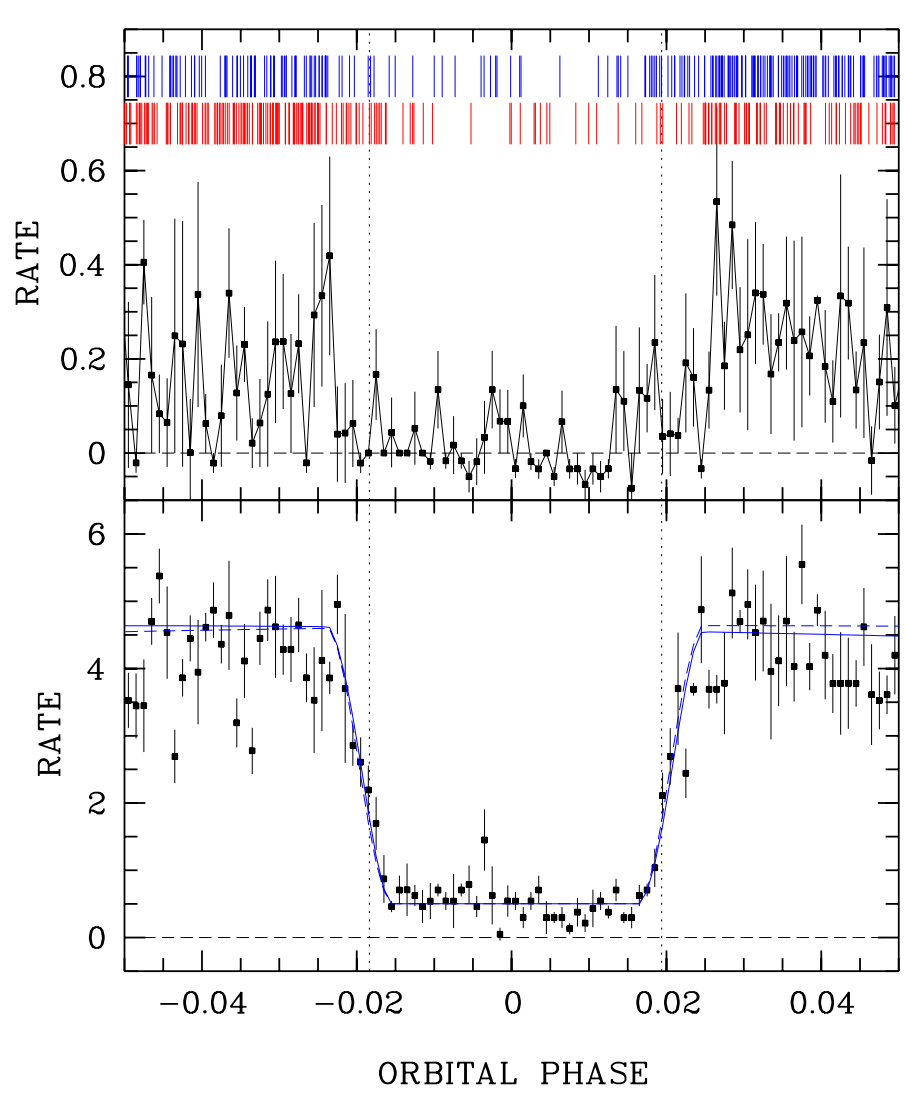

Fig. 6. Eclipse profile of OV Boo at X-ray (top) and UV (bottom) energies. The original data from EPIC-pn and the OM were binned with $4 \mathrm{~s}$ time resolution and phase-averaged using 1000 phase bins per orbital cycle. The dashed vertical lines indicate the eclipse length of $151 \mathrm{~s}$ measured from the OM data shifted by $2 \mathrm{~s}$ toward a later phase in agreement with the updated ephemeris (see Fig. 2). The vertical blue ticks in the upper panel indicate arrivals of individual photons (pn, MOS1, and $\operatorname{MOS} 2$ ); the red ticks are the same events reflected about $\phi=0.0$ (corrected for the $2 \mathrm{~s}$ offset) to highlight asymmetries in the X-ray eclipse. The curves in the lower panel shown in blue are WD plus spot models whose parameters are explained in the text.

system by varying the metal abundance of the two-temperature plasma component. We found that the fit favors a low metallicity, but this parameter also becomes degenerate with the temperature of the hotter MEKAL. In other words, reducing the metallicity caused the temperature to run to an unphysically high value, and both parameters ended up with uncertainties larger than their values: for a metallicity of 0.12 the warm MEKAL's best fit temperature is $30 \pm 30 \mathrm{keV}$ and for 0.07 it has reached the code hard limit of $79.9 \mathrm{keV}$. We therefore conclude that there is weak but inconclusive evidence in the X-ray data that OV Boo is metal poor, but for the fits we report we have kept the MEKAL values at their default solar metallicity value.

Extrapolating the luminosity measurement to bolometric luminosity is usually a questionable procedure, but since we have little absorption and no significant soft blackbody component, probably safe enough in this case. Using the cflux command we found that this model gives an unabsorbed bolometric luminosity of $\sim 5 \times 10^{30} \mathrm{erg} \mathrm{s}^{-1}$, about $40 \%$ greater than the luminosity in the $0.2-10.0 \mathrm{keV}$ range reported in Table 2. This equates to a mass accretion rate of $\sim 4 \times 10^{-13} \mathrm{M}_{\odot} \mathrm{yr}^{-}$using the WD parameters given above.

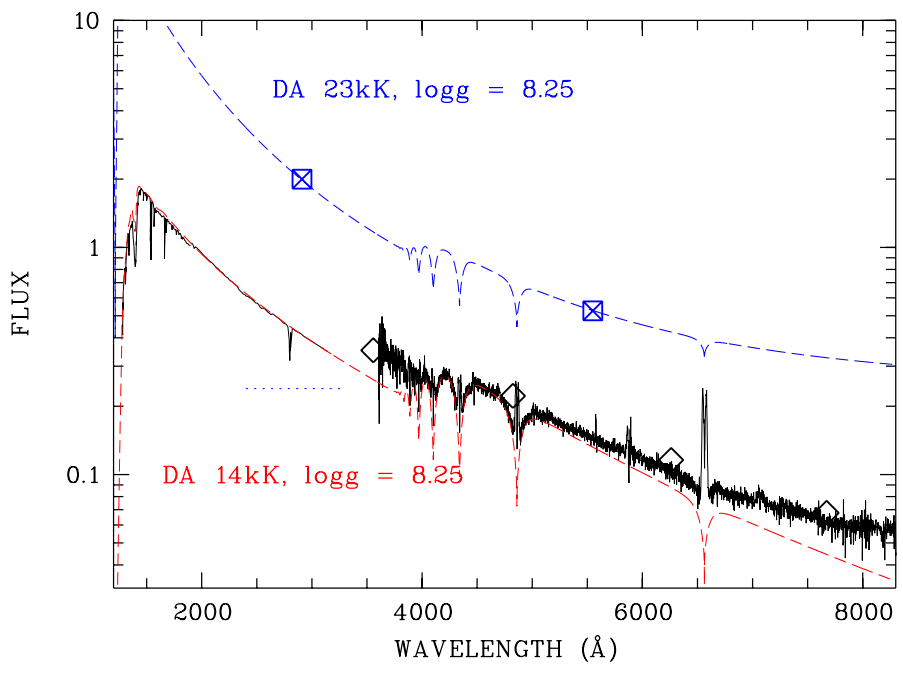

Fig. 7. Optical to UV spectral energy distribution of OV Boo. Shown are the pre-outburst spectra from the HST (Uthas et al.2011) and the SDSS, and pre-outburst SDSS ugri photometric data, together with postoutburst photometric data from the OM and AAVSO (this work). All observed pre-outburst data are shown in black, the two post-outburst data points in blue. White dwarf model spectra for 14 and $23 \mathrm{kK}$, are shown in red and blue, respectively. Flux units are $10^{-15} \mathrm{erg} \mathrm{cm}^{-2} \mathrm{~s}^{-1} \AA^{-1}$. The $23 \mathrm{kK}$ spectrum was shifted downwards by 0.239 units to account for the non-WD part of the spectrum, whose brightness is indicated by the short blue dashed line at $3000 \AA$.

\subsection{Spot model and optical/UV spectral energy distribution}

In Figure 6 we show the eclipse profile of OV Boo at X-ray and UV wavelengths from our observations with XMM-Newton. The upper panel shows the phase-folded EPIC-pn light curve.

Apart from the two-second offset of the eclipse center, the UV eclipse profile is symmetric, but it is clear that the $\mathrm{X}$ ray eclipse leads the formal eclipse midpoint by approximately 0.0035 phase units, or around 15 seconds. To highlight the asymmetry of the X-ray eclipse, the arrival times of X-ray photons from all three EPIC cameras are indicated by vertical lines, both in original time sequence (shown in blue) and mirrored at phase zero (in red), taking the two-second offset into account. We denote the four contact points of the eclipse of the WD $t_{1} \ldots t_{4}$. The X-ray ingress coincides with the first contact point, $t_{1}$, while the initial egress happens about $10 \mathrm{~s}$ before $t_{3}$. The X-ray egress seems to be complete at $t_{4}$.

The UV eclipse ingress and egress lasts about $31 \pm 11 \mathrm{~s}$, which is compatible with the size of the WD. At the bottom of the eclipse the flux stays finite at about $10 \%$ of the out-ofeclipse level. There is a gradual decline in the UV flux through the eclipse. We proceed by assuming that all the eclipsed UV flux originates from the WD. The non-eclipsed flux must originate from the accretion disk or structure therein (hot spot). We neglect the variability of the non-eclipsed part, and model the UV data by applying a geometrical model that assumes a WD (and optionally an accretion-heated spot on it) that is gradually obscured and released by the donor star. A grid of pure hydrogen atmosphere models was folded through the response curves of the UV filter. The models used are those of Koester (2010) for pure hydrogen atmospheres and $\log g=8.25$. The choice of the gravity has little impact on the continuum brightness which is the only relevant quantity obtained from the spectral models for our light curve model. A circular spot was placed on the WD and was characterized by its position, its extent, and its temperature distribution. A linear temperature decrease was assumed from 
a maximum in the spot center to the temperature of the undisturbed WD. The WD surface was divided in small tiles and each tile was assigned a certain brightness according to the chosen model. The binary model then determines which tiles are visible at any given phase. Their summed brightness, modified by foreshortening and limb darkening, then gives a predicted signal as a function of binary phase.

Since the data appeared rather noisy, several important parameters were pre-defined. The distance was assumed to be $210 \mathrm{pc}$. The parameters of the WD (mass, radius) and the inclination $\left(=83.6^{\circ}\right)$ were those of Littlefair et al. (2007). The temperature also needs to be pre-defined since the data, shown in Fig. 4 display some flare-like excursions which make it difficult to derive the $T_{\mathrm{WD}}$ from fits to the UV light curve. We therefore fixed the temperature of the WD by analyzing the optical-UV spectral energy distribution shown in Fig. 7] It shows pre-outburst data from the HST and the SDSS (both the spectrum and the ugriz photometry) and the post-outburst OM and AAVSO photometry (mean bright phase level). For the presentation in Fig. 7] we use Koester's spectra, which are available online (Koester 2010. The pre-outburst data from the UV to the optical are compatible with the $14,000 \mathrm{~K} \mathrm{DA}$ WD model spectrum derived by Uthas et al. (2011). The model gives an excellent representation of the UV spectra; it underpredicts the observed $u$-band flux from the SDSS and also falls short in the near-infrared. However, such deviations are thought to be of minor relevance in the current context. The excess emission observed post-outburst at optical (AAVSO) and UV (OM) wavelengths can consistently be attributed to a heated WD of about $23,000 \mathrm{~K}$ (blue symbols and blue curve in Fig. 7). A corresponding WD model with uniform temperature of $23,000 \mathrm{~K}$ give an acceptable representation of the observed data. The uncertainty is estimated to be on the order of $500 \mathrm{~K}$.

Using a homogeneous WD with a temperature of $23,000 \mathrm{~K}$ also gives an excellent representation of the detailed eclipse shape in the UV (see Fig. 6). The motivation to also account for a spot with enhanced temperature was derived from enhanced emission around the eclipse. Similar models were used in the past; a graphical illustration of the visibility of such a heated spot is given by Gänsicke et al. (1998), among others. Given the low $\mathrm{S} / \mathrm{N}$ of the data and the high parameter degeneracy, we fixed the spot location and half-opening angle $\left(20^{\circ}\right)$ and searched for a spot temperature $T_{\text {spot }}$ such that the summed signal of the spot and the undisturbed WD is compatible with the overall light curve of Fig. 4. We found that $T_{\text {spot }}=27,000 \mathrm{~K}$ gives a satisfactory explanation of the data. The model curves shown in blue in Figs. 4 and 6 were computed for azimuth values of $20^{\circ}$ (pointing into the quadrant where the accretion stream leaving $L_{1}$ lies, solid line) and of $-10^{\circ}$ (dashed line), which lies behind the binary meridian. The different parameters cannot be distinguished in the data with high phase resolution centered on the eclipse (Fig. 6), but only in the total light curve. The model with an azimuth of $-10^{\circ}$ was motivated by the phase of the X-ray hump, whereas the model with $+20^{\circ}$ was motivated by the location of the UV hump.

In summary, the optical to UV spectral energy distribution and the detailed shape of the eclipse observed with the OM can be nicely explained with a homogeneously heated WD of $23,000 \mathrm{~K}$, about $9,000 \mathrm{~K}$ hotter than during quiescence. Some residual UV emission at the bottom of the WD eclipse at a 5\% level must have an origin somewhere away from the WD (i.e., in the accretion disk).

\footnotetext{
${ }^{2}$ http://svo2.cab.inta-csic.es/theory/newov2/index.php?models=koester2 riods of high background. The phase-folded UV and X-ray light
}

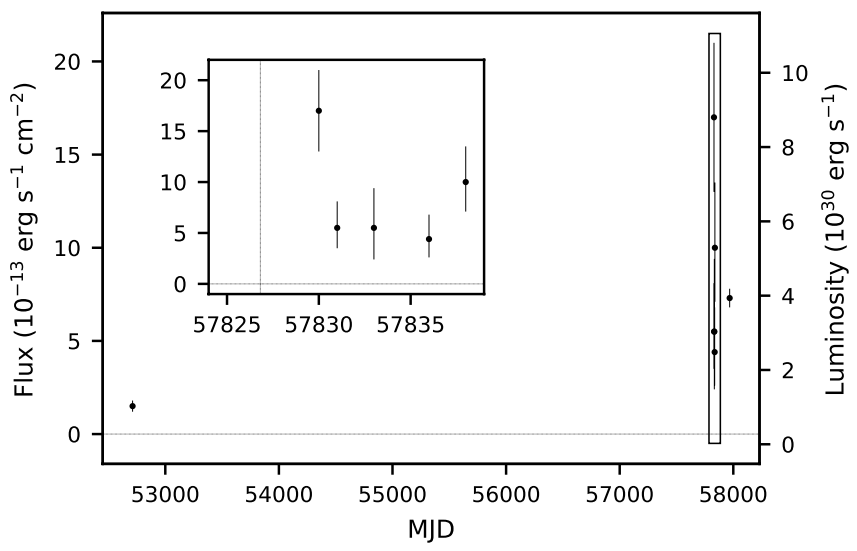

Fig. 8. Long-term $X$-ray light curve of OV Boo in the $0.2-10 \mathrm{keV}$ energy range. The Chandra and XMM-Newton observations correspond to the first and last points, respectively. The Swift observations of the DN outburst are shown in the inset. Error bars are at $1 \sigma$ significance. The luminosity has been calculated using the $210 \mathrm{pc}$ distance; the relative uncertainty is about $7 \%$. The vertical line in the inset indicates the time of the first outburst optical observation.

\subsection{X-ray emission in the eclipse}

We generated an EPIC-pn eclipse image using only photons in the phase interval $(-0.012:+0.012)$ and the energy interval $E=0.2-5.0 \mathrm{keV}$ with a total exposure of 412 seconds. Source detection at a fixed position using the task edetect_stack yields $5.1 \pm 3.1$ photons or a rate of $0.0123 \pm 0.0075$ at a $D E T \_M L$ of only 2 , consistent with a non-detection. Using the SAS-task eupper we determine a $3 \sigma$ upper limit count rate in the eclipse of $0.042 \mathrm{~s}^{-1}$. This is converted into flux by an absorbed plasma $F_{\text {ecl }}<5 \times 10^{-14} \mathrm{erg} \mathrm{cm}^{-2} \mathrm{~s}^{-1}$ or an upper limit luminosity of $L_{\text {ecl }}<3 \times 10^{29} \mathrm{erg} \mathrm{s}^{-1}$. The sensitivity is not sufficient to address the question of X-ray emission from the donor star.

\subsection{Long-term X-ray behavior}

The entire long-term X-ray light curve of OV Boo is shown in Figure 8, it comprises data obtained with Chandra, Swift, and $\mathrm{XMM}-$ Newton, obtained in quiescence long before the DN outburst, during the outburst, and in the immediate post-outburst phase. We have omitted the three shortest Swift exposures because the source was not detected. The maximum flux observed was about $1.6 \times 10^{-12} \mathrm{erg} \mathrm{cm}^{-2} \mathrm{~s}^{-1}$.

The Chandra observation was $4.9 \mathrm{ks}$ long and delivered 24 photons in a 10" source extraction region, for a backgroundsubtracted source count rate of $4.2_{-0.9}^{+1.1} \mathrm{cts} \mathrm{ks}^{-1}$. There were not spectral shape as in the XMM-Newton observation we infer an absorbed flux of around $1-1.5 \times 10^{-13} \mathrm{erg} \mathrm{s}^{-1} \mathrm{~cm}^{-2}$, slightly higher than the catalog flux, but around 5-6 times fainter than in the XMM-Newton observation.

\section{Analysis and results: SDSSJ1035}

SDSSJ1035 was very faint during the $23.8 \mathrm{ks}$ XMM-Newton observation. Only 215,20 , and 54 photons were detected from the source by the EPIC- $p n$, MOS1, and MOS2 cameras, respectively. Fortunately, the entire observation was unaffected by peemission model with $1 \mathrm{keV}$ and $N_{\mathrm{H}}=1 \times 10^{20} \mathrm{~cm}^{-2}$ and yields enough photons to extract a spectrum, but assuming the same 


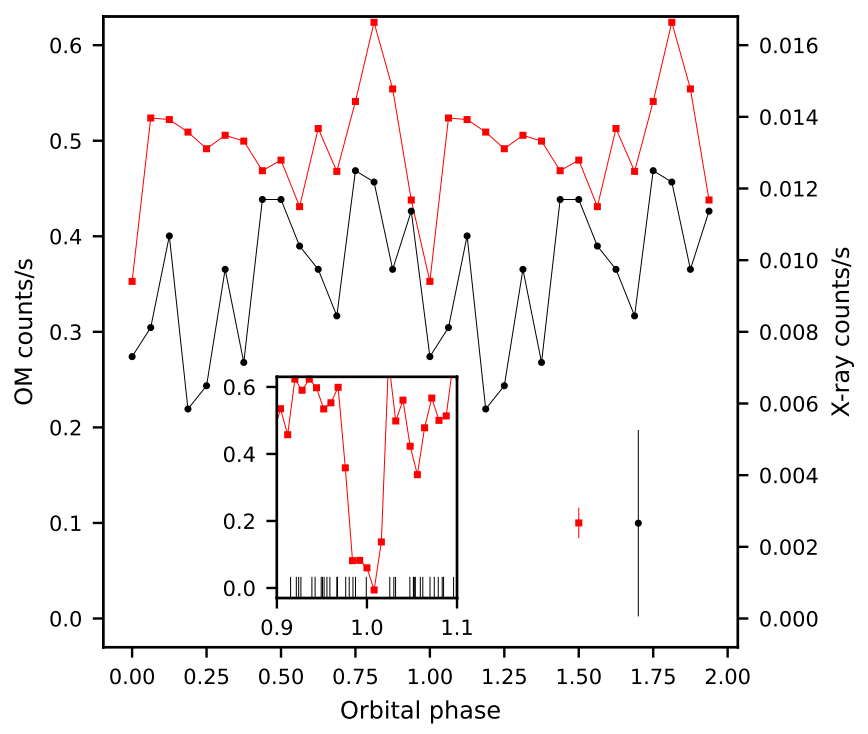

Fig. 9. Phase-folded UV (red squares) and X-ray (black circles) light curve of SDSSJ1035 obtained with XMM-Newton. The light curve has been duplicated for clarity. Mean $1 \sigma$ uncertainties are indicated by the symbols below the curves in the lower right corner. The inset shows the region around the eclipse, with finer binning for the UV points. To avoid cluttering the graph we omit the uncertainties. The vertical black lines indicate the arrival time of the $\mathrm{X}$-ray photons.

curves are shown in Figure 9 We used the $82.1 \mathrm{~min}$ period of Littlefair et al. (2006) and shifted the phase so that the eclipse, which is clearly visible in the UV data, coincides with $\phi=0$. The first eclipse in the UV data occurs at approximately BJD $(\mathrm{TDB})=2,451,521.8636(1)$.

The eclipses are clearly visible in the phase-folded EPIC-pn data as well, but the data are poor quality and there is a second downward excursion that is probably not real, so we cannot draw a firm conclusion on the existence of an eclipse in the X-ray data. These data were sufficient to extract coarse spectra for the $p n$ and MOS 2 cameras, and produced a single bin for MOS1. These are shown in Figure 10. We grouped these to a minimum of 16 counts per bin between 0.2 and $10 \mathrm{keV}$.

Despite the low spectral resolution we were able to determine that a single-temperature MEKAL spectrum did not fit the data well: $\chi_{v}^{2}=1.5$ (12). Formally, this has a chance likelihood of $10 \%$, but obvious residuals at the low-energy end suggest a poor model fit. A two-temperature plasma was a much better fit. No absorption was necessary, likely owing to the low counts. The best fit parameters are summarized in Table 3 . The spectrum could be described with the combination of a hotter (about $2 \mathrm{keV}$ ) collisionally ionized plasma and a cooler component. The cooler component could be described either with a plasma component (parameters listed in Tab. 3) or with a blackbody, and cannot be statistically discerned. The cool component could be the sought for (or expected) emission from the optically thick boundary layer. Using the observed integrated flux in the 0.2 - $10 \mathrm{keV}$ energy band, the implied instantaneous mass accretion for the 0.94 solar mass WD with $R_{\mathrm{WD}}=0.0087 \mathrm{R}_{\odot}$ is only $8 \times 10^{-15} \mathrm{M}_{\odot} \mathrm{yr}^{-1}$ (Littlefair et al. 2006).

SDSSJ1035 was not detected in X-rays in either of the Swift observations. This is unsurprising; if the source had the same spectral shape and intensity as in the XMM-Newton observation, Swift would have detected at most one or two photons in either of its observations.
Table 3. Spectral fit for SDSSJ1035. Fluxes and luminosities are in the $0.2-10 \mathrm{keV}$ range, and uncertainties are $1 \sigma$.

\section{Parameter Value}

\begin{tabular}{ll}
\hline $\mathrm{kT}$ & $1.7_{-0.3}^{+0.6 \mathrm{keV}}$ \\
norm & $6.5_{-1.6}^{+1.8} \times 10^{-6}$ \\
$\mathrm{kT}$ & $100^{+50} \mathrm{eV}$ \\
norm & $8.1_{-5.5}^{+11.9} \times 10^{-6}$ \\
\hline Flux & $2.0 \pm 0.2 \times 10^{-14} \mathrm{erg} \mathrm{s}^{-1} \mathrm{~cm}^{-2}$ \\
Lum & $1.0 \pm 0.2 \times 10^{29} \mathrm{erg} \mathrm{s}^{-1}$ \\
\hline$\chi_{v}^{2}$ & $0.78(10)$
\end{tabular}

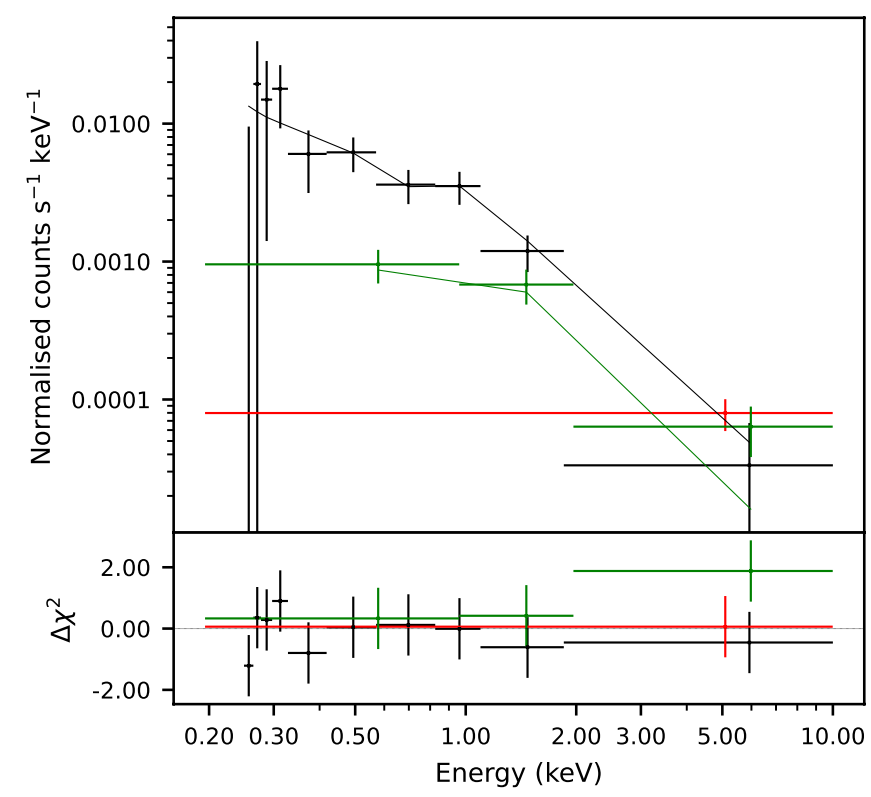

Fig. 10. X-ray spectrum (points with error bars) and fitted twotemperature plasma model (solid lines) of SDSSJ1035. EPIC-pn, MOS1, and MOS2 are plotted in black, red, and green, respectively.

The optical data from the SDSS and the UV data for this source recorded by XMM-Newton, Swift, and the Galaxy Evolution Explorer (GALEX; Martin et al. 2005) are shown in Figure 11. The effective wavelengths of the filters are given in Page et al. (2014) (for XMM-Newton), Poole et al. (2008) (for Swift), and Morrissey et al. (2007) (for GALEX). For the OM we derived two data points, one at the orbital minimum, which represents the maximum contribution of the WD, and one at the orbital maximum, which is due to emission from the hot spot on the accretion disk rim. Part of the variability seen with Swift might be due to sampling the light curve at different orbital phases; on the other hand, the source appeared generally around 0.5 magnitudes brighter in 2018 than it was in 2012 in all filters, which probably indicates a real overall brightness increase.

Littlefair et al. (2008) derive $T_{\text {eff }}=10100 \pm 200 \mathrm{~K}$ and a distance of $171 \pm 10$ pc for SDSSJ1035. The revised Gaia distance of 209 pc requires a parameter update for the WD. Assuming the WD radius (hence mass) is to be fixed by the detailed eclipse model, an increase in $T_{\text {eff }}$ is required. We found a model with $T_{\text {eff }}=11250 \pm 250 \mathrm{~K}$ that gives the measured WD flux from the deconvolution by Littlefair et al. (2008), and that was adequate to reflect the optical-UV spectral energy distribution and to match, not overpredict, the minimum flux observed by Swift-UVOT. The rather moderate increase in $T_{\text {eff }}$ nevertheless 


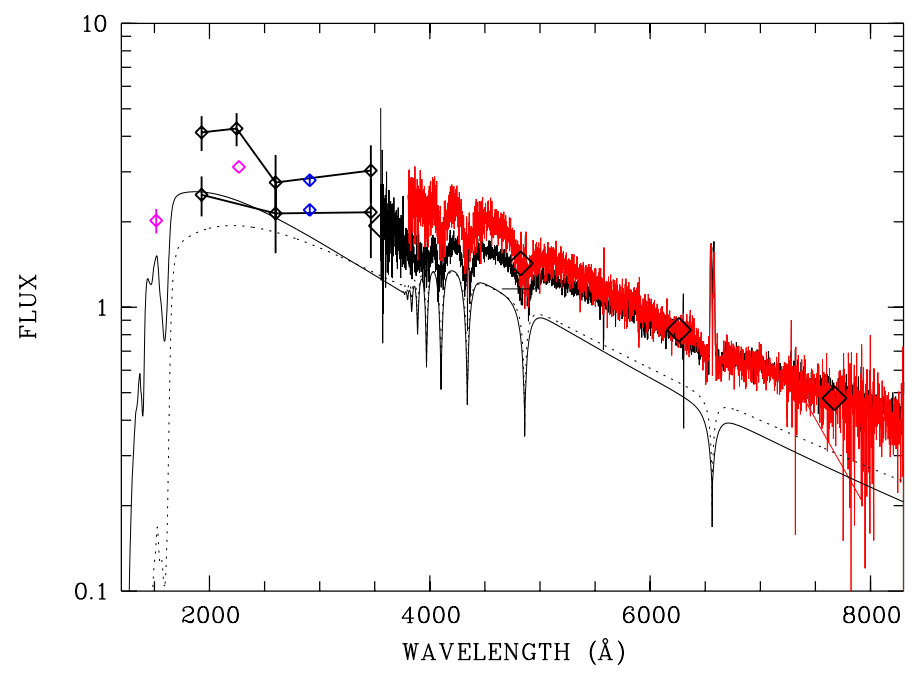

Fig. 11. Optical to UV spectral energy distribution of SDSSJ1035. Shown are the two spectra obtained by the SDSS (black and red) plus SDSS photometry (black diamonds), Swift-UVOT photometry (black diamonds), GALEX FUV and NUV data (magenta diamonds), and the XMM-Newton OM observations obtained by us at orbital minimum and maximum (blue diamonds). Swift data obtained at the same epoch are connected with lines. The black solid line is a $\log g=8.25$, $T_{\text {eff }}=11500 \mathrm{~K} \mathrm{DA} \mathrm{WD} \mathrm{model} \mathrm{spectrum} \mathrm{scaled} \mathrm{to} \mathrm{a} \mathrm{distance} \mathrm{of} 209 \mathrm{pc}$. Flux units are $10^{-16} \mathrm{erg} \mathrm{cm}^{-2} \mathrm{~s}^{-1} \AA^{-1}$.

means a significant increase (about $60 \%$ ) in the implied longterm mass accretion rate (from about $1 \times 10^{-11} \mathrm{M}_{\odot} \mathrm{yr}^{-1}$ to about $1.6 \times 10^{-11} \mathrm{M}_{\odot} \mathrm{yr}^{-1}$, as inferred by using Eq. 2 in Townsley \& Gänsicke 2009).

\section{Discussion}

We analyzed X-ray, ultraviolet, and optical observations of two eclipsing period-bouncing CVs (i.e., CVs with a degenerate donor star): OV Boo and SDSSJ1035. Both objects were discovered at X-ray wavelengths indicating ongoing accretion. OV Boo shows an X-ray eclipse, which hints at an emission region close to the WD, likely the boundary layer. The situation is less clear in SDSSJ1035 due to its weaker X-ray signal.

Although the two stars are at about the same distance, OV Boo was much brighter at X-ray and UV wavelengths thanks to a higher instantaneous accretion rate and a recent (rare) DN outburst. The DN outburst happened 142 days before our observations with XMM-Newton were scheduled. These data gave deeper insight into the origin of the X-ray and UV emission through a detailed eclipse analysis than those of SDSSJ1035, and are discussed first in the following paragraphs.

The main findings for OV Boo can be summarized as follows. The X-ray and UV light curves outside the eclipse are not completely flat but seem to show some flickering behavior and some excess emission centered around phase zero. Apart from the flickering, the detailed UV eclipse light curve and the mean out-of-eclipse brightness are compatible with a WD with $T_{\text {eff }}=23 \mathrm{kK}$, some $9000 \mathrm{~K}$ hotter than found before the DN outburst. Both the UV and the optical magnitude found contemporaneously are compatible with this temperature. Approximately $10 \%$ of the UV flux does not go into eclipse. This remaining flux displays some decrease through the eclipse. It appears possible that this remaining flux originates from the hot spot where the accretion stream hits the outer rim of the accretion disk. The detailed eclipse models by Littlefair et al. (2007) suggest that the total eclipse of the hot spot begins at phase 0.1 , which does not seem to comply with our data. We do not want to overinterpret our data, but we cannot exclude some extra emission at this phase from another location in the binary.

The X-ray eclipse seemingly has a faster ingress and egress than the UV eclipse. In addition, the eclipse center seems to be shifted in phase to occur earlier by 0.0035 phase units or about $15 \mathrm{~s}$. While the eclipse ingress seems to be sharp, the egress may be structured and happen in two steps. The mean X-ray spectrum is highly absorbed, a factor of 15 higher than the galactic column density in the direction of the star, and is purely thermal. Taking these findings together a picture emerges in which all the observed X-ray emission is located in the boundary layer (BL) between the inner rim of the disk and the WD surface (see the thorough discussion of quiescence DN X-ray emission in Mukai 2017). The high column density suggests that any potential optically thick emission from the BL is absorbed and not accessible to us. Some extra absorption by matter (e.g., at the hot spot) might be responsible for the early ingress. This matter is no longer in the line of sight at eclipse egress so that this feature is observed at the expected time and/or phase.

The implied instantaneous mass accretion rate from the XMM-Newton observations is low, $\sim 4 \times 10^{-13} \mathrm{M}_{\odot} \mathrm{yr}^{-1}$, but still a factor of 5-6 higher than during a serendipitous Chandra observation taken a few years before the outburst. Whether the mass transfer rate from the donor was higher than normal or the mass flow rate through the disk still enhanced in the post-outburst phase cannot be determined from our data.

We could derive an upper limit for the X-ray emission in the eclipse of $L_{\mathrm{ecl}}<3 \times 10^{29} \mathrm{erg} \mathrm{s}^{-1}$. We are not reaching sufficient sensitivity to constrain X-ray emission from the donor star.

SDSSJ1035 is much fainter than OV Boo, and therefore difficult to study. It is less luminous by a factor of $\sim 40$ in both X-rays and UV. The instantaneous accretion rate of SDSSJ1035 is lower than that of OV Boo by around 1.5 orders of magnitude (a factor of about 50 ), $8 \times 10^{-15} \mathrm{M}_{\odot} \mathrm{yr}^{-1}$. This rate is even lower than that of the only magnetic period bouncer studied by Stelzer et al. (2017) who found a mass accretion rate of $3.2 \times 10^{-14} \mathrm{M}_{\odot} \mathrm{yr}^{-1}$.

The two objects have the same inclination of about $83^{\circ}$. OV Boo shows a high level of intrinsic absorption, whereas SDSSJ1035 does not show any sign of absorption in its X-ray spectrum, indicating rather different shapes of the accretion disk and hot spots on its rim. Instead of absorption SDSSJ1035 shows some sign of the expected soft emission from the boundary layer, but its faintness does not allow us to derive conclusive evidence on its existence.

Through the inclusion of the UV in our analysis we were able to derive revised temperatures for the WDs in both objects. The high temperature found in our observation in OV Boo is due to the recent DN outburst, the long-term average temperature of the WD is better described with the value of $14,000 \mathrm{~K}$ found by Uthas et al. (2011). The larger distance to J1035 together with the various photometric data obtained in the UV led to the revised $T_{\text {eff }}$ of $11,250 \mathrm{~K}$. The updated WD temperatures together with their precisely determined masses can be used to infer the long-term mass accretion rates for both objects. Using Eq. 2 of Townsley \& Gänsicke (2009) the rates are $\log \dot{M}=-10.33 \mathrm{M}_{\odot} \mathrm{yr}^{-1}$ and $-10.79 \mathrm{M}_{\odot} \mathrm{yr}^{-1}$ for $\mathrm{OV}$ Boo and J1035, respectively. These values place the two objects outside the tracks of Willems et al. (2005) that were used by Littlefair et al. (2008) to discuss the evolutionary status of these shortperiod objects. Whether this deviation is significant for the class needs to be seen through an enlarged sample of objects. 


\section{Conclusion}

This last section is devoted to a quick discussion of the prospect of enlarging the sample through identification programs of the $\mathrm{X}$-ray surveys with eROSITA. eROSITA has just completed its first X-ray all-sky survey (Predehl et al.2020), eRASS1. The first survey reached a point-source limiting flux in the soft band (0.2$2.3 \mathrm{keV}$ ) of $5 \times 10^{-14} \mathrm{erg} \mathrm{cm}^{-2} \mathrm{~s}^{-1}$, the final depths after completion of the planned eight independent surveys in the ecliptic equatorial regions will be $1.1 \times 10^{-14} \mathrm{erg} \mathrm{cm}^{-2} \mathrm{~s}^{-1}$. If we assume that the flux of J1035 is representative of the period bouncing, low-luminosity $\mathrm{CVs}$ at $210 \mathrm{pc}$, then the radii to search for more objects of this kind as X-ray emitters in the first and the full eROSITA all sky survey are 133 and $280 \mathrm{pc}$, respectively. How many bouncers can we detect in the corresponding volumes? The volume surveyed in eRASS1 corresponds to the $150 \mathrm{pc}$ sample surveyed by Pala et al. (2020) who only have three candidate period bouncers in their sample of 42 objects (with a possible missing 12 objects of any CV subtype for $100 \%$ completeness). Predicted space densities of Belloni et al. (2020) (their model 324509 in Table 1) for period bouncers are as high as $\rho_{0}=17 \times 10^{-6} \mathrm{~cm}^{-3}$ with a factor of two uncertainty. In agreement with this, Hernández Santisteban et al. (2018) derive a $2 \sigma$ upper limit for period bouncing objects they call dead CVs of $\rho_{0}=2 \times 10^{-5} \mathrm{~cm}^{-3}$. Using a constant density, justified by the expected large scale-height of more than $400 \mathrm{pc}$ for this old population, and the given upper limit we expect a maximum of 24 objects in a volume of radius $150 \mathrm{pc}$ and about 180 in a volume with radius $280 \mathrm{pc}$, to be reached after finishing the eROSITA all-sky surveys.

For the two objects studied here we determined the flux ratio between the optical and the X-ray flux in quiescence to check the feasibility of eROSITA follow-up. For OV Boo we use $g=18.31, f_{\mathrm{X}}=1 \times 10^{-13} \mathrm{erg} \mathrm{cm}^{-2} \mathrm{~s}^{-1}$ (Chandra), for J1035 we use $g=18.79$ and $f_{\mathrm{X}}=2 \times 10^{-14} \mathrm{erg} \mathrm{cm}^{-2} \mathrm{~s}^{-1}$ to obtain flux ratios $\log \left(f_{\mathrm{X}} / f_{\text {opt }}\right)=\log f_{\mathrm{X}}-0.4 \mathrm{mag}+5.37$ of -0.3 and -0.8 , respectively. These numbers are close to those found for ordinary CVs, and should help to separate accreting objects from coronal emitters. Very late-type stars at the bottom of the main sequence may reach similarly high values, but will be distinguished from the low-luminosity CVs through their distinct red color as opposed to the very blue color of the period bouncers (see Comparat et al.2020, their Fig. 11). At the given flux limit all candidates will have counterparts in the Gaia catalogs. Hence, the feasibility to build statistically meaningful samples through comprehensive X-ray identification programs is excellent.

Acknowledgements. We thank an anonymous referee for carefully reading the original manuscript and giving useful advice. This work was supported by the German DLR under contracts 50 OR 1405, 50 OR 1711, 50 OR 1814, and 50 OX 1901. We acknowledge with thanks the variable and comparison star observations from the AAVSO International Database contributed by observers worldwide and used in this research. Many of the AAVSO observations were the result of Centre for Backyard Astronomy (CBA) campaigns. We thank the AAVSO observers who made available their data via the AAVSO and used here to refine the orbital period: David Cejudo Fernandez and Lew Cook from Spain and the US. This research has made use of the APASS database, located at the AAVSO web site. Funding for APASS has been provided by the Robert Martin Ayers Sciences Fund. This work has made use of Astropy (Astropy Collaboration et al. 2013, 2018). This research has made use of data, software and/or web tools obtained from the High Energy Astrophysics Science Archive Research Center (HEASARC), a service of the Astrophysics Science Division at NASA/GSFC and of the Smithsonian Astrophysical Observatory's High Energy Astrophysics Division.

\section{References}

Arnaud, K. A. 1996, in Astronomical Society of the Pacific Conference Series, Vol. 101, Astronomical Data Analysis Software and Systems V, ed. G. H. Jacoby \& J. Barnes, 17

Astropy Collaboration, Price-Whelan, A. M., Sipőcz, B. M., et al. 2018, ArXiv e-prints [arXiv: 1801.02634]

Astropy Collaboration, Robitaille, T. P., Tollerud, E. J., et al. 2013, A\&A, 558, A33

Bailer-Jones, C. A. L., Rybizki, J., Fouesneau, M., Mantelet, G., \& Andrae, R. 2018, ArXiv e-prints, arXiv: 1804.10121

Belloni, D., Schreiber, M. R., Pala, A. F., et al. 2020, MNRAS, 491, 5717

Bengtsson, H. 2017a, vsnet-alert, 20792, 1

Bengtsson, H. 2017b, vsnet-alert, 20795, 1

Blackburn, J. K. 1995, in Astronomical Society of the Pacific Conference Series, Vol. 77, Astronomical Data Analysis Software and Systems IV, ed. R. A. Shaw, H. E. Payne, \& J. J. E. Hayes, 367

Burleigh, M. R., Marsh, T. R., Gänsicke, B. T., et al. 2006, MNRAS, 373, 1416 Burrows, D. N., Hill, J. E., Nousek, J. A., et al. 2005, Space Sci. Rev., 120, 165 Comparat, J., Merloni, A., Dwelly, T., et al. 2020, A\&A, 636, A97

den Herder, J. W., Brinkman, A. C., Kahn, S. M., et al. 2001, A\&A, 365, L7

Drake, A. J., Djorgovski, S. G., Mahabal, A., et al. 2009, ApJ, 696, 870

Gaia Collaboration, Brown, A. G. A., Vallenari, A., et al. 2018, ArXiv e-prints [arXiv: 1804.09365]

Gaia Collaboration, Prusti, T., de Bruijne, J. H. J., et al. 2016, A\&A, 595

Gänsicke, B. T., Hoard, D. W., Beuermann, K., Sion, E. M., \& Szkody, P. 1998, A\&A, 338, 933

Goliasch, J. \& Nelson, L. 2015, ApJ, 809, 80

Hernández Santisteban, J. V., Knigge, C., Pretorius, M. L., Sullivan, M., \& Warner, B. 2018, MNRAS, 473, 3241

HI4PI Collaboration, Ben Bekhti, N., Flöer, L., et al. 2016, A\&A, 594, A116

Kirsch, M. G. F., Altieri, B., Chen, B., et al. 2004, in Society of Photo-Optical Instrumentation Engineers (SPIE) Conference Series, Vol. 5488, UV and Gamma-Ray Space Telescope Systems, ed. G. Hasinger \& M. J. L. Turner, 103-114

Koester, D. 2010, Mem. Soc. Astron. Italiana, 81, 921

Littlefair, S. P., Dhillon, V. S., Marsh, T. R., et al. 2007, MNRAS, 381, 827

Littlefair, S. P., Dhillon, V. S., Marsh, T. R., et al. 2008, MNRAS, 388, 1582

Littlefair, S. P., Dhillon, V. S., Marsh, T. R., et al. 2006, Science, 314, 1578

Martin, D. C., Fanson, J., Schiminovich, D., et al. 2005, ApJ, 619, L1

Masci, F. J., Laher, R. R., Rusholme, B., et al. 2019, PASP, 131, 018003

Mason, K. O., Breeveld, A., Much, R., et al. 2001, A\&A, 365, L36

Mewe, R., Gronenschild, E. H. B. M., \& van den Oord, G. H. J. 1985, A\&AS, 62, 197

Morrissey, P., Conrow, T., Barlow, T. A., et al. 2007, ApJS, 173, 682

Mukai, K. 2017, PASP, 129, 062001

Page, M. J., Yershov, V., Breeveld, A., et al. 2014, in Proceedings of Swift: 10 Years of Discovery (SWIFT 10), held 2-5 December 2014 at La Sapienza University, Rome, Italy., 37

Pala, A. F., Gänsicke, B. T., Breedt, E., et al. 2020, MNRAS, 494, 3799

Patterson, J., de Miguel, E., Barret, D., et al. 2017, Society for Astronomical Sciences Annual Symposium, 36, 1

Patterson, J., Thorstensen, J. R., \& Knigge, C. 2008, PASP, 120, 510

Poole, T. S., Breeveld, A. A., Page, M. J., et al. 2008, MNRAS, 383, 627

Predehl, P., Andritschke, R., Arefiev, V., et al. 2020, arXiv e-prints, arXiv:2010.03477

Reis, R. C., Wheatley, P. J., Gänsicke, B. T., \& Osborne, J. P. 2013, MNRAS, 430, 1994

Savoury, C. D. J., Littlefair, S. P., Dhillon, V. S., et al. 2011, MNRAS, 415, 2025 Schwope, A. D. \& Christensen, L. 2010, A\&A, 514, A89

Schwope, A. D. \& Thinius, B. D. 2014, Astronomische Nachrichten, 335, 357

Southworth, J., Gänsicke, B. T., Marsh, T. R., et al. 2006, MNRAS, 373, 687

Stelzer, B., de Martino, D., Casewell, S. L., Wynn, G. A., \& Roy, M. 2017, A\&A, 598, L6

Strüder, L., Briel, U., Dennerl, K., et al. 2001, A\&A, 365, L18

Szkody, P., Henden, A., Fraser, O. J., et al. 2005, AJ, 129, 2386

Tanabe, K., Akazawa, H., \& Fukuda, N. 2018, Information Bulletin on Variable Stars, 6251, 1

Townsley, D. M. \& Gänsicke, B. T. 2009, ApJ, 693, 1007

Turner, M. J. L., Abbey, A., Arnaud, M., et al. 2001, A\&A, 365, L27

Uthas, H., Knigge, C., Long, K. S., Patterson, J., \& Thorstensen, J. 2011, MNRAS, 414, L85

Willems, B., Kolb, U., Sandquist, E. L., Taam, R. E., \& Dubus, G. 2005, ApJ, 635,1263 


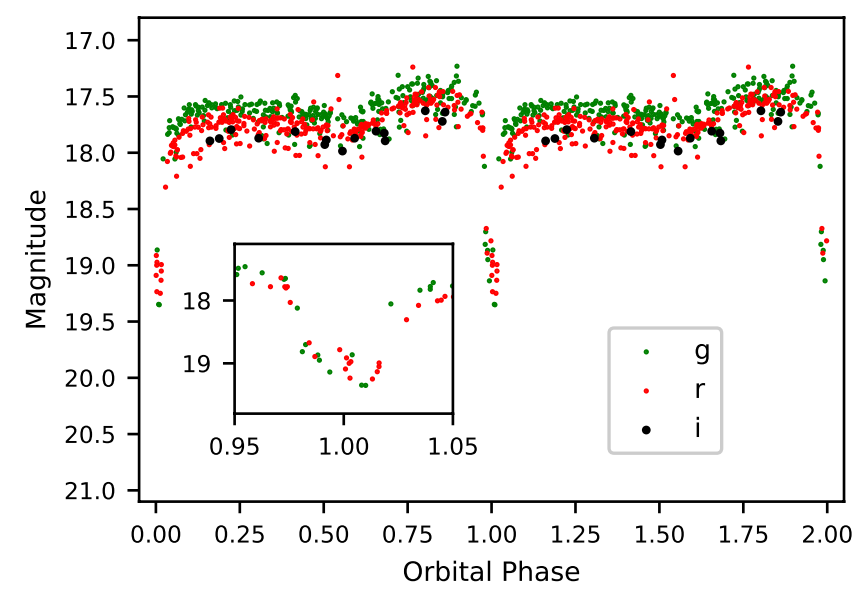

Fig. A.1. Phase-folded light curve of OV Boo from the ZTF (2018 March - 2019 June). The light curve has been duplicated for clarity. The inset shows the $r$ and $g$ data in the region of the eclipses.

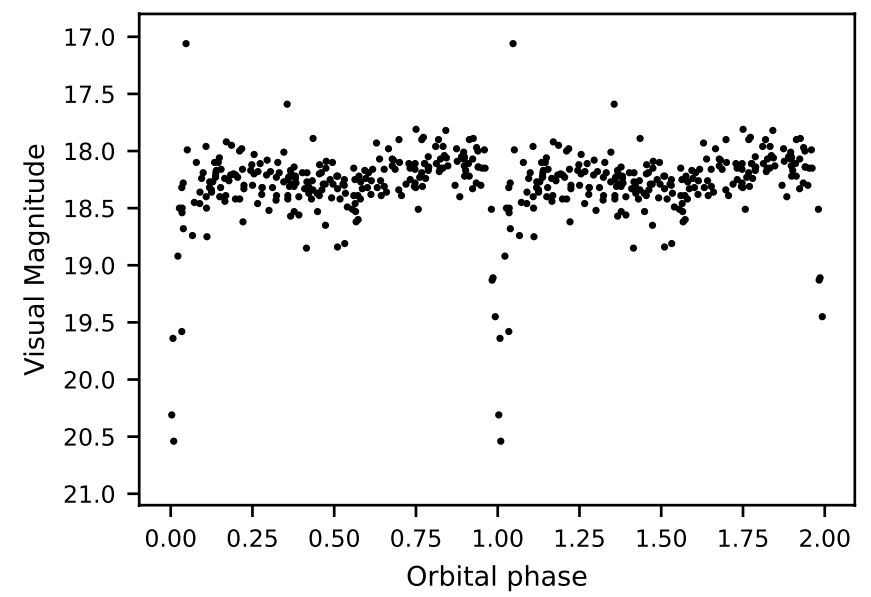

Fig. A.2. Phase-folded light curve of OV Boo from CSS (2007-2013). The light curve has been duplicated for clarity.

\section{Appendix A: Eclipse times used to derive the ephemeris of OV Boo}


Table A.1. Eclipse midpoint timings for OV Boo. Listed are the cycle number, the times of eclipse center in Barycentric Julian Days in the TT time system (terrestrial time), the uncertainty of the timing, the deviation from the linear ephemeris given in Eq. 22, and an observer code. Observer codes P, L, H, A, and OM stand for Patterson et al. (2008), Littlefair et al. (2007), Hubble, the AAVSO, and the Optical Monitor on XMM-Newton, respectively.

\begin{tabular}{|c|c|c|c|c|c|c|c|c|c|}
\hline Cycle & $\mathrm{BJD}(\mathrm{TDB})$ & Unc. (s) & $\mathrm{O}-\mathrm{C}(\mathrm{s})$ & Obs. & Cycle & $\mathrm{BJD}(\mathrm{TDB})$ & Unc. (s) & $\mathrm{O}-\mathrm{C}(\mathrm{s})$ & Obs. \\
\hline 0 & 2453498.89301 & 11.0 & -1.9 & $\mathrm{P}$ & 8234 & 2453879.78421 & 11.0 & 1.4 & $\mathrm{P}$ \\
\hline 1 & 2453498.93924 & 11.0 & -4.3 & $\mathrm{P}$ & 8236 & 2453879.87667 & 11.0 & -3.5 & $\mathrm{P}$ \\
\hline 2 & 2453498.98548 & 11.0 & -5.9 & $\mathrm{P}$ & 8237 & 2453879.92298 & 11.0 & 1.0 & $\mathrm{P}$ \\
\hline 82 & 2453502.68616 & 11.0 & -4.8 & $\mathrm{P}$ & 8238 & 2453879.96925 & 11.0 & 2.0 & $\mathrm{P}$ \\
\hline 83 & 2453502.73244 & 11.0 & -2.9 & $\mathrm{P}$ & 8254 & 2453880.70934 & 11.0 & -1.8 & $\mathrm{P}$ \\
\hline 84 & 2453502.77868 & 11.0 & -4.5 & $\mathrm{P}$ & 8255 & 2453880.75560 & 11.0 & -1.6 & $\mathrm{P}$ \\
\hline 85 & 2453502.82495 & 11.0 & -3.5 & $\mathrm{P}$ & 8256 & 2453880.80185 & 11.0 & -2.4 & $\mathrm{P}$ \\
\hline 86 & 2453502.87121 & 11.0 & -3.3 & $\mathrm{P}$ & 8257 & 2453880.84814 & 11.0 & 0.4 & $\mathrm{P}$ \\
\hline 87 & 2453502.91745 & 11.0 & -4.9 & $\mathrm{P}$ & 8258 & 2453880.89434 & 11.0 & -4.7 & $\mathrm{P}$ \\
\hline 88 & 2453502.96374 & 11.0 & -2.2 & $\mathrm{P}$ & 8275 & 2453881.68082 & 11.0 & 3.0 & $\mathrm{P}$ \\
\hline 107 & 2453503.84263 & 1.1. & -3.8 & $\mathrm{P}$ & 8276 & 2453881.72704 & 11.0 & -0.3 & $\mathrm{P}$ \\
\hline 108 & 2453503.88887 & 11.0 & -5.4 & $\mathrm{P}$ & 8277 & 2453881.77332 & 11.0 & 1.5 & $\mathrm{P}$ \\
\hline 109 & 2453503.93514 & 11.0 & -4.4 & $\mathrm{P}$ & 8278 & 2453881.81953 & 11.0 & -2.6 & $\mathrm{P}$ \\
\hline 129 & 2453504.86031 & 11.0 & -4.1 & $\mathrm{P}$ & 8365 & 2453885.84401 & 11.0 & -2.3 & $\mathrm{P}$ \\
\hline 130 & 2453504.90656 & 11.0 & -4.8 & $\mathrm{P}$ & 8366 & 2453885.89027 & 11.0 & -2.1 & $\mathrm{P}$ \\
\hline 131 & 2453504.95282 & 11.0 & -4.7 & $\mathrm{P}$ & 8367 & 2453885.93657 & 11.0 & 1.5 & $\mathrm{P}$ \\
\hline 622 & 2453527.66565 & 11.0 & -5.9 & $\mathrm{P}$ & 16710 & 2454271.86984 & 11.0 & -3.6 & $\mathrm{P}$ \\
\hline 623 & 2453527.71193 & 11.0 & -4.0 & $\mathrm{P}$ & 16711 & 2454271.91614 & 11.0 & -0.0 & $\mathrm{P}$ \\
\hline 624 & 2453527.75819 & 11.0 & -3.9 & $\mathrm{P}$ & 16731 & 2454272.84120 & 11.0 & -0.6 & $\mathrm{P}$ \\
\hline 625 & 2453527.80446 & 11.0 & -2.9 & $\mathrm{P}$ & 16732 & 2454272.88754 & 11.0 & -2.2 & $\mathrm{P}$ \\
\hline 626 & 2453527.85071 & 11.0 & -3.6 & $\mathrm{P}$ & 16752 & 2454273.81273 & 11.0 & -0.2 & $\mathrm{P}$ \\
\hline 627 & 2453527.89697 & 11.0 & -3.5 & $\mathrm{P}$ & 16753 & 2454273.85901 & 11.0 & 1.7 & $\mathrm{P}$ \\
\hline 752 & 2453533.67928 & 11.0 & -1.9 & $\mathrm{P}$ & 37774 & 2455246.25550 & 2.0 & -3.4 & $\mathrm{H}$ \\
\hline 753 & 2453533.72555 & 11.0 & -0.9 & $\mathrm{P}$ & 37775 & 2455246.30177 & 2.0 & -2.4 & $\mathrm{H}$ \\
\hline 754 & 2453533.77177 & 11.0 & -4.2 & $\mathrm{P}$ & 37777 & 2455246.39429 & 2.0 & -2.1 & $\mathrm{H}$ \\
\hline 774 & 2453534.69693 & 11.0 & -4.8 & $\mathrm{P}$ & 96081 & 2457943.44054 & 18.0 & 2.3 & $\mathrm{~A}$ \\
\hline 775 & 2453534.74318 & 11.0 & -5.6 & $\mathrm{P}$ & 96103 & 2457944.45822 & 18.0 & 2.0 & A \\
\hline 776 & 2453534.78944 & 11.0 & -5.4 & $\mathrm{P}$ & 96124 & 2457945.42942 & 18.0 & -17.5 & A \\
\hline 777 & 2453534.83577 & 11.0 & 0.8 & $\mathrm{P}$ & 96146 & 2457946.44741 & 18.0 & 9.0 & A \\
\hline 6482 & 2453798.739589 & 0.7 & 0.1 & $\mathrm{~L}$ & 96167 & 2457947.41877 & 18.0 & 3.4 & $\mathrm{~A}$ \\
\hline 6500 & 2453799.572252 & 0.7 & 1.2 & $\mathrm{~L}$ & 96168 & 2457947.46511 & 18.0 & 10.4 & A \\
\hline 6502 & 2453799.664756 & 0.7 & 0.1 & $\mathrm{~L}$ & 96174 & 2457947.74253 & 18.0 & -0.8 & $\mathrm{~A}$ \\
\hline 6521 & 2453800.543664 & 0.7 & 0.1 & $\mathrm{~L}$ & 96175 & 2457947.78892 & 18.0 & 10.6 & A \\
\hline 6524 & 2453800.682427 & 0.7 & -0.9 & $\mathrm{~L}$ & 96189 & 2457948.43646 & 18.0 & 3.9 & A \\
\hline 6569 & 2453802.764065 & 0.7 & 0.2 & $\mathrm{~L}$ & 96210 & 2457949.40788 & 18.0 & 3.5 & A \\
\hline 6586 & 2453803.550464 & 0.7 & 0.8 & $\mathrm{~L}$ & 96211 & 2457949.45424 & 18.0 & 12.3 & $\mathrm{~A}$ \\
\hline 6587 & 2453803.596708 & 0.7 & -0.5 & $\mathrm{~L}$ & 96232 & 2457950.42553 & 18.0 & 0.6 & A \\
\hline 8213 & 2453878.81274 & 11.0 & -2.5 & $\mathrm{P}$ & 96644 & 2457969.48401 & 4.2 & 4.4 & $\mathrm{OM}$ \\
\hline 8214 & 2453878.85902 & 11.0 & -0.6 & $\mathrm{P}$ & 96645 & 2457969.53024 & 4.2 & 2.0 & $\mathrm{OM}$ \\
\hline 8215 & 2453878.90526 & 11.0 & -2.2 & $\mathrm{P}$ & 96646 & 2457969.57649 & 4.2 & 1.3 & $\mathrm{OM}$ \\
\hline 8216 & 2453878.95153 & 11.0 & -1.2 & $\mathrm{P}$ & 96647 & 2457969.62275 & 4.2 & 1.2 & $\mathrm{OM}$ \\
\hline
\end{tabular}

